\title{
Genome Analysis of Antimicrobial Resistance Genes and Virulence Factors in Multidrug-Resistant Campylobacter fetus Subspecies Isolated from Sheath Wash
}

\author{
Mpinda Edoaurd Tshipamba* ${ }^{1}$, Ngoma Lubanza ${ }^{1}$ and Mulunda Mwanza ${ }^{1}$ \\ ${ }^{I}$ Department of Animal Health, School of Agriculture, Faculty of Agriculture, Science and Technology, Mafikeng Campus, North-West University, \\ Private Bag X2046, Mmabatho, 2735, South Africa \\ *Corresponding author's Email: edotshipamba@gmail.com; (DORCiD: 0000-0003-4469-3200
}

\begin{abstract}
Campylobacter fetus subspecies are mostly characterized by reproductions problems in cattle and sheep. This study aimed to study the genetic profile and assess the genes mechanism of resistance and their virulence factors using genome sequence analysis. A total of 59 confirmed Campylobacter fetus subspecies based on molecular assays and DNA sequencing were subjected to antimicrobial susceptibility test against 14 antibiotic agents representing the five classes of antibiotics using the disc diffusion method. In addition, sequencing the genome of all strains induced complete resistance against all tested antibiotics. The results of the antimicrobial test indicated that $54.4 \%$ had a resistance profile, $26.3 \%$ were intermediate, while $19.3 \%$ were observed to be susceptible. The Whole Genome Sequencing (WGS) result revealed the presence of different genes, such as Broad-specificity multidrug efflux pump and 16S rRNA (guanine ${ }^{527}-\mathrm{N}^{7}$ )-methyltransferase (gidB), efflux pump conferring antibiotic resistance (MacA and $\mathrm{MacB})$, protein-altering cell wall charge conferring antibiotic resistance (PgsA), which have never been reported in Campylobacter fetus subspecies. The WGS also revealed the presence of genes that involved in colonization, adhesion, motility, and invasion, such as type IV secretion system protein (VirD4), S-Layer, cytolethal distending toxin (A, B, and C), Campylobacter invasion antigen (CiaB), and fic domain protein (fic) were among important CDS. The presence of these uncommon genes explains the resistance of Campylobacter fetus subspecies against different tested antibiotics. The results of this study can be used to implement molecular surveillance of Campylobacter fetus subspecies and conduct further studies on the resistance mechanism in these subspecies.
\end{abstract}

Keywords: Broad-specificity multidrug efflux pump, Campylobacter fetus subspecies, Genome analysis, Methyltransferase gidB, Multidrug resistance.

\section{INTRODUCTION}

Embryonic deaths, temporary infertility with mild endometritis, and abortions can be caused by Campylobacter fetus subspecies in the herd, which resulted in substantial production losses and economic hardship for producers worldwide (Bellows et al., 2002; Morrell et al., 2011). Venereal diseases such as bovine genital campylobacterosis are considered as the primary cause of reproductive loss (McCool et al., 1988). Experimental studies revealed that treating carrier bulls has been done by victimization, either vaccination with a monovalent oil-based immunizing agent or recurrent antimicrobial agent. Furthermore, several of the revealed antimicrobial treatment protocols that used antimicrobial agents are not commercially obtainable. What is more, it had been confirmed that none of the revealed studies had provided robust proof supporting a treatment of choice that eliminates shedding in infected bulls (Vasquez et al., 1983; Truyers et al., 2014).

On the other hand, researchers discovered that vaccination itself is not ample to manage a pestilence. However, habitually testing and culling of infected bulls is suggested. Testing and culling procedures have a considerable price related to the premature loss of high price animals; particularly, considering the value of breeding bulls (Guerra et al., 2014; Erickson et al., 2017). A study conducted on the analysis of long action oxytetracycline and a monovalent immunizing agent for the management of Campylobacter fetus subsp. venerealis disease in bulls ended that there have been no vital variations between treated and untreated bulls. No matter the diagnostic applied, treatment with two label doses of this regime failed to stop the shedding of Campylobacter fetus subsp. venerealis in treated bulls, therefore, is not suggested as an efficient management strategy (Erickson et al., 2017). The resistance against long action oxytetracycline observed by Erickson et al. (2017) may be correlated with the previous findings obtained by Mak et al. (2014), that researchers indicated that bacteria species contain a variable number of resistance mechanisms against 
different antimicrobial agents for their self-defense to ensure their complete protection. This resistance mechanism is, for the most part, co-regulated through the expression of resistance genes (Mak et al., 2014).

Thus considering the importance of Campylobacter fetus subspecies relatedness to food, public, and animal health and their resistance profile to different antibiotics, this study aimed to study the genetic profile and assess genes mechanism of resistance and their virulence factors by using genome sequence analysis.

\section{MATERIALS AND METHODS}

\section{Ethical approval}

Ethical consideration for this study was submitted and approved by the Animal Production Sciences Research Ethics Committee of the North-West University (NWU-AnimProdRec), South Africa. The study procedures were performed in accordance with the requisite ethical standards.

\section{Study design and area}

This was a cross-sectional descriptive study conducted in Dr. Ruth Mompati District Municipality in North-West Province, South-Africa, from January 2018 to December 2019, focusing on positive Campylobacter fetus subspecies collected from the state veterinary services and processed at the North-West University Laboratory at Mafikenf Campus for further analysis.

\section{Bacterial identification}

Positive Campylobacter fetus subspecies isolated from sheath wash and obtained from bulls that were from in the North-West province in South Africa were transported in Steve medium was spread plated on the surface of Skirrow's agar (Tryptose blood Agar Base, Oxoid, UK) mixed with 7\% sheep blood and supplemented with Campylobacter selective supplement (Skirrow, SR0069E, Oxoid, England). Plates were then incubated anaerobically at $37^{\circ} \mathrm{C}$ for 72 hours using an anaerobic Jar 2.5 L (Oxoid, England) containing CampyGenTM sachet CN0025A (Oxoid, England) to produce a microaerophilic atmospheric condition for the growth of Campylobacter fetus. All the plates were firstly examined for growth after the 72 hours incubation period, and if growth was not observed, the plates were re-incubated for a further 48 hours. After the incubation period, all the plates were subjected to morphological identification (a small colony, mucoid, greyish, flat colony with irregular edges). The suspected plates of Campylobacter fetus were considered for further analysis (Acke et al., 2009).

\section{Molecular assays for the identification of Campylobacter fetus subspecies}

\section{Genomic DNA extraction}

The extraction of genomic DNA was carried out according to the standard molecular methods (Di Giannatale et al., 2014) using Zymo-Research Kit (Bio-Lab, South Africa) following the manufacturer's instructions. The extracted genomic DNA was then quantified spectrophotometrically using a Nanodrop® ND-1000. The genomic DNA was kept in fresh DNA tubes to serve as a DNA template for subsequent PCR.

\section{Polymerase chain reaction}

The confirmation of members of the genus Campylobacters fetus subsp in this study was performed using Polymerase Chain Reaction (PCR). A total volume of $50 \mu \mathrm{L}$ containing $20 \mu \mathrm{L}$ PCR 2xMaster Mix (Bio-Labs, Iso 9001, England), $4 \mu \mathrm{L}$ template DNA, $22 \mu \mathrm{L}$ nuclease DNA free water (Bio-concept 1td, ISO 9001, paradiesrain 14, Switzerland) and $4 \mu \mathrm{L}$ of oligonucleotide primer (Forward and Reverse) were prepared and mixed in PCR tubes. The DNA was amplified using a Thermal cycler (Bio-Rad, T100 ${ }^{\mathrm{TM}}$ Thermal cycler, Model: T100 ${ }^{\mathrm{TM}}$ Thermal cycler, serial number: 621BR19028, Singapore) as it has been presented in table 1 (Schulze et al., 2006; Wangroongsarb et al., 2011).

\section{The DNA sequencing analysis}

The amplified PCR products of isolated bacteria were sent to Inqaba Biotechnology (Pretoria, South Africa) for sequencing. The sequences and chromatograms were observed with Bio-systems. Forward and reverse sequences were compared and corrected for conformity. Blast program tools were applied to search for the sequences (Altschul et al., 1997) to find the closest match for each one in the Gen-Bank. The closest sequences were then downloaded and aligned with the original sequences using clustal and edited using Finch TV version 1.4.0. 


\section{Analysis of the antimicrobial profile of Campylobacter fetus subspecies}

The evaluation of the antimicrobial profile of Campylobacter fetus in this study was carried out using the KirbyBauer disc diffusion method (BAKWS and Turck, 1966; Washington and Wood, 1995). The results were interpreted based on the guidelines of the Clinical and Laboratory Standards Institute guidelines (institute, 2013). Multiple drug resistance was defined as resistance to two or more classes of antimicrobials tested. The antibiotics panel selected for screening Campylobacter fetus subspecies were the one mostly used in the case of campylobacteriosis in both veterinary and human medicine. The following 14 antibiotics were used: Erythromycin (15 mg), Azithromycin (15 mg), Streptomycin (10mg), Neomycin (30 mg), Gentamicin (10 mg), Ampicillin (10 mg), Amoxicillin (10 mg), Ciprofloxacin (5 mg), Enrofloxacin (5 mg), Norfloxacin (5 mg), Doxycycline (5 mg), Tetracycline (30 mg), Chloramphenicol (30 mg) and Nalidixic Acid (30 mg).

\section{Genome sequencing, assembly, and annotation}

The extracted genome DNA samples were fragmented using an enzymatic approach (NEB Ultra II FS Kit, BioLabs, England). The DNA fragmented results were sized selected (200-500 bp), using AMPure XP beads, the fragments were end-repaired, and Illumina specific adapter sequences were ligated to each fragment. Each sample was individually indexed, and a second size selection step was performed. Samples were then quantified, using a fluorometric method, diluted to a standard concentration $(4 \mathrm{nM})$, and then sequenced on Illumina's NextSeq platform, using a NextSeq 300 cycle kit, following a standard protocol per manufacturer's instruction to generate a total number of 43,826,295 reads and 41,141,918 reads with 2X 300bp paired-end read length and the coverage was 40X respectively for Campylobacter fetus subsp venerealis NW_ME2 and Campylobacter fetus subsp. fetus NW_ME1.

The sequenced data generated were analyzed using appropriate bioinformatics tools, with default parameters, through the Kbase platform (Arkin et al., 2018). The sequenced data was filtered for low-quality reads and adapter regions using Trimmomatic- v0.36 (Bolger et al., 2014), and Fast QC-version 0.11.5 was employed to assess the quality of data. The genome assembly was performed using SPAdes- version 3.13.0 (Bankevich et al., 2012). The functional annotation of the entire draft assembly was carried out with the NCBI Prokaryotic Genome Annotation Pipeline (PGAP) (Haft et al., 2018) and Rapid Annotations using Subsystems Technology (RAST, version 11) (Aziz et al., 2008).

\section{Statistical analysis}

Data were analyzed using SPSS (version 23.0). Descriptive statistics such as frequencies and percentages were applied to determine the occurrence of bacteria and the antimicrobial profile of the isolated bacteria. Pearson's chisquare test of association was used to ascertain the association between the area and occurrence of bacteria and antimicrobial profile. Furthermore, the study used the Kruskal Wallis test and the Mann-Whitney's U to ascertain whether the resistance differed significantly across the antibiotics and whether the resistance differed across the bacterial species. For significant tests, cross-tabulations (Tables 2 and 3) were used to explain the association of the difference in resistance against different antibiotic profile of the isolated bacteria. Based on Kruskal Wallis test results, the p-value $<0.05$ was considered significant, and they have been presented in tables 4,5 , and 6 .

Table 1. Primers and PCR conditions

\begin{tabular}{|c|c|c|}
\hline Primers & Conditions & References \\
\hline $\begin{array}{l}\text { MG3F (5'-GGTAGCCGCAGCTGCTAAGAT-3') } \\
\text { MG4R (5'-TAG CTACAA TAA CGA CAA CT-3'). }\end{array}$ & $\begin{array}{l}\text {-Initial denaturation: } 95^{\circ} \mathrm{C} \text { for } 15 \text { seconds } \\
\text {-Second denaturation: } 96^{\circ} \mathrm{C} \text { for } 15 \text { seconds } \\
\text { - Annealing: } 60^{\circ} \mathrm{C} \text { for } 60 \text { seconds } \\
\text { - Extension: } 72^{\circ} \mathrm{C} \text { for } 90 \text { seconds } \\
\text { The processes of the second denaturation, annealing, and } \\
\text { extension were repeated for } 35 \text { cycles and a final extension } \\
\text { done at } 180^{\circ} \mathrm{C} \text { for } 10 \mathrm{~min}\end{array}$ & (Schulze et al., 2006) \\
\hline $\begin{array}{l}\text { CFCH57F (GCAAGTCGAACGGAGTATTA) } \\
\text { CF1054R (GCAGCACCTGTCTCAACT) }\end{array}$ & $\begin{array}{l}\text {-Initial denaturation: } 94^{\circ} \mathrm{C} \text { for } 5 \text { minutes } \\
\text { - Second denaturation: } 94^{\circ} \mathrm{C} \text { for } 60 \text { seconds } \\
\text { - Annealing at } 50^{\circ} \mathrm{C} \text { for } 60 \mathrm{sec} \\
\text { - Extension: } 72^{\circ} \mathrm{C} \text { for } 60 \mathrm{~seconds} \\
\text { - The processes of the second denaturation, annealing, and } \\
\text { extension were repeated for } 30 \text { cycles and a final extension } \\
\text { done at } 720 \mathrm{C} \text { for } 10 \mathrm{~min}\end{array}$ & $\begin{array}{l}\text { (Wangroongsarb et al., } \\
\text { 2011). }\end{array}$ \\
\hline
\end{tabular}


Table 2. The occurrence of antimicrobial profile of Campylobacter fetus subsp. fetus isolated from sheath wash

\begin{tabular}{|c|c|c|c|c|}
\hline \multirow{2}{*}{ Bacteria } & \multirow{2}{*}{ Antibiotics } & \multicolumn{3}{|c|}{ Resistance } \\
\hline & & Resistant & Intermediate & Susceptible \\
\hline \multirow{15}{*}{ Campylobacter fetus subsp. fetus } & Tetracycline & $59.3 \%$ & $29.6 \%$ & $11.1 \%$ \\
\hline & Doxycycline & $44.4 \%$ & $44.4 \%$ & $11.1 \%$ \\
\hline & Ampicillin & $63.0 \%$ & $29.6 \%$ & $7.4 \%$ \\
\hline & Amoxicillin & $59.3 \%$ & $29.6 \%$ & $11.1 \%$ \\
\hline & Erythromycin & $25.9 \%$ & $48.1 \%$ & $25.9 \%$ \\
\hline & Azithromycin & $38.5 \%$ & $34.6 \%$ & $26.9 \%$ \\
\hline & Neomycin & $57.7 \%$ & $19.2 \%$ & $23.1 \%$ \\
\hline & Streptomycin & $48.1 \%$ & $33.3 \%$ & $18.5 \%$ \\
\hline & Gentamicin & $44.4 \%$ & $25.9 \%$ & $29.6 \%$ \\
\hline & Ciprofloxacin & $63.0 \%$ & $22.2 \%$ & $14.8 \%$ \\
\hline & Nalidixic acid & $85.2 \%$ & $11.1 \%$ & $3.7 \%$ \\
\hline & Norfloxacin & $33.3 \%$ & $29.6 \%$ & $37.0 \%$ \\
\hline & Enrofloxacin & $37.0 \%$ & $33.3 \%$ & $29.6 \%$ \\
\hline & Chloramphenicol & $51.9 \%$ & $37.0 \%$ & $11.1 \%$ \\
\hline & Total & $50.8 \%$ & $30.6 \%$ & $18.6 \%$ \\
\hline
\end{tabular}

Table 3. Phenotypic antimicrobial profile of Campylobacter fetus subsp. venerealis isolated from sheat wash

\begin{tabular}{|c|c|c|c|c|}
\hline \multirow{2}{*}{ Bacteria } & \multirow{2}{*}{ Antibiotics } & \multicolumn{3}{|c|}{ Cross-tabulation of the antimicrobial profile } \\
\hline & & Resistant & Intermediate & Susceptible \\
\hline \multirow{15}{*}{ Campylobacter fetus subsp. venerealis } & Tetracycline & $71.0 \%$ & $25.8 \%$ & $3.2 \%$ \\
\hline & Doxycycline & $54.8 \%$ & $32.3 \%$ & $12.9 \%$ \\
\hline & Ampicillin & $67.7 \%$ & $16.1 \%$ & $16.1 \%$ \\
\hline & Amoxicillin & $58.1 \%$ & $22.6 \%$ & $19.4 \%$ \\
\hline & Erythromycin & $48.4 \%$ & $16.1 \%$ & $35.5 \%$ \\
\hline & Azithromycin & $51.6 \%$ & $22.6 \%$ & $25.8 \%$ \\
\hline & Neomycin & $64.5 \%$ & $32.3 \%$ & $3.2 \%$ \\
\hline & Streptomycin & $71.0 \%$ & $16.1 \%$ & $12.9 \%$ \\
\hline & Gentamicin & $48.4 \%$ & $29.0 \%$ & $22.6 \%$ \\
\hline & Ciprofloxacin & $51.6 \%$ & $19.4 \%$ & $29.0 \%$ \\
\hline & Nalidixic acid & $61.3 \%$ & $22.6 \%$ & $16.1 \%$ \\
\hline & Norfloxacin & $45.2 \%$ & $29.0 \%$ & $25.8 \%$ \\
\hline & Enrofloxacin & $45.2 \%$ & $19.4 \%$ & $35.5 \%$ \\
\hline & Chloramphenicol & $67.7 \%$ & $12.9 \%$ & $19.4 \%$ \\
\hline & Total & $57.6 \%$ & $22.6 \%$ & $19.8 \%$ \\
\hline
\end{tabular}

\section{RESULTS}

\section{Antimicrobial resistance profile of Campylobacter fetus subspecies}

The overall results of the antimicrobial resistance profile revealed that $54.4 \%$ of the isolated bacteria indicated a resistance profile against the antimicrobial tested, $26.3 \%$ presented intermediate profile, while a small portion of isolated bacteria $19.3 \%$ were observed to susceptible as they have been demonstrated in figure 1 .

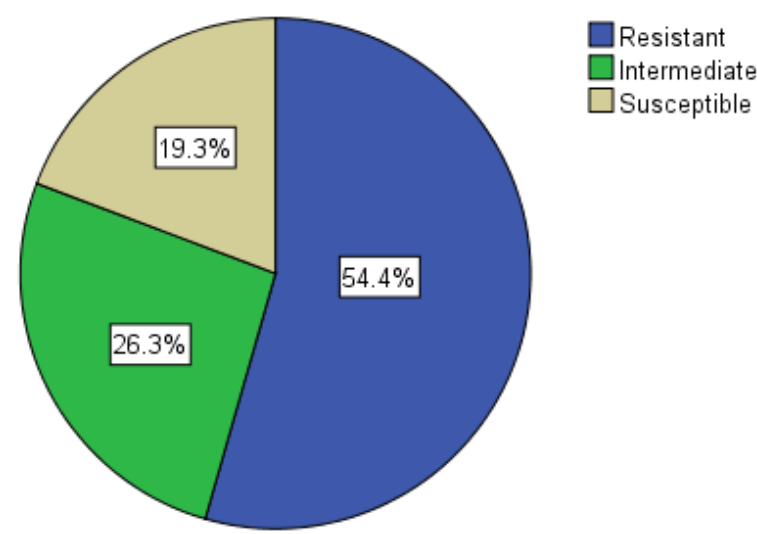

Figure 1. Overall profiles of Campylobacter fetus subspecies subjected to the antimicrobial test 


\section{The occurrence of antimicrobial resistance profile of Campylobacter fetus subspecies}

The analysis revealed that the antibiotic resistance of the Campylobacter fetus subsp fetus differed across the different antibiotics. For instance, it was observed that more strains of this subspecies were resistant to nalidixic acid $(85.2 \%)$ than those that were resistant to ampicillin or ciprofloxacin (63\%), as presented in table 2. Some strains of these subspecies were found to be more susceptible to norfloxacin (37\%) than those that were susceptible to gentamicin (29.6\%). It was also observed that $71.0 \%$ of Campylobacter fetus subsp.venerealis were resistant to tetracycline, $71.0 \%$ streptomycin, while $61.3 \%$ were resistant to nalidixic acid, $67.7 \%$ were resistant to chloramphenicol, and $35.5 \%$ of the isolates were found to be susceptible to erythromycin (Table 3).

\section{Relationship between resistance profile and antibiotics}

Pearson Chi-square test indicated that they were no association between the area from where samples were collected and the occurrences of isolated bacteria $(\mathrm{p}>0.05)$ as presented in table 5. The Kruskal Wallis test results indicated that the antibiotic resistance of Campylobacter fetus subsp. fetus differed significantly in accordance with the different tested antibiotics $(\mathrm{p}<0.05)$. However, there was no significant difference in the antibiotic resistance across the different antibiotics for the Campylobacter fetus subsp. venerealis ( $\mathrm{p}>0.05)$, as presented in table 4 . The MannWhitney's $U$ test presented that the antibiotic resistance to nalidixic acid differed significantly across the two bacterial species $(\mathrm{p}<0.05)$ as presented in table 6.

\section{Encoding of resistance genes in Campylobacter fetus subspecies using genome sequencing}

The analysis revealed numerous genes involved in the resistance and virulence, as presented in tables 7 and 8 . Additionally, it was observed that the present genome Campylobacter fetus subsp. fetus NW_ME1 contained six mobile element proteins, Campylobacter fetus subsp. venerealis_NW_ME2 was observed to have nine mobile element proteins. It was also observed that genes MurA (UDP-N-acetylglucosamine 1-carboxyvinyltransferase), Dxr (1-deoxy-D-xylulose 5-phosphate reductoisomerase) were exclusively found in Campylobacter fetus subsp. fetus NW_ME1. Furthermore, the encoded genes were located at different positions of the DNA strand and had a different DNA length, as is demonstrated in figures 2 and 7.

\section{General genome feature and data availability}

Data from our genome shotgun project of Campylobacter fetus subsp. venerealis NW_ME2 and Campylobacter fetus subsp. fetus NW_ME1 have been deposited at DDBJ/ENA/Gene Bank under the accession numbers JAATTN000000000 and JAAVIZ000000000. The version published in this paper was JAATTN010000000 and JAAVIZ010000000. The Raw reads were also submitted in NCBI/ SRA and are available in NCBI under Bio project PRJNA614613 and PRJNA614609. The genome of Campylobacter fetus subsp. venerealis NW_ME2 has a total length of $1,891,894$ bp with an average $\mathrm{G}+\mathrm{C}$ content of $33.2 \%$, while Campylobacter fetus subsp. fetus NW_ME1 has a total length of $1,845,025$ nucleotides with a GC content of $33.4 \%$. Both isolates harbor resistance genes, and virulence factors were demonstrated in figures 8 and 9 . The nucleotide sequences with high similarities ( $\geq 95 \%)$ were also submitted in the NCBI Gene bank and they are available under these accession numbers: MT138642; MT138643; MT138644; MT138645; MT138646; MT138647; MT138648; MT138649; MT138650; MT138651; MT138652; MT138653; MT138654; MT138655; MT138656; MT138657; MT138658; MT138659; MT138660; MT138661

\section{Pathogenomics and phylogenetic analysis}

The Pathogenomics analysis using Pathogen Finder version 1.1 revealed that our input organism Campylobacter fetus subsp. fetus NW_ME1 was predicted to be a human pathogen. The probability of being a human pathogen was 0.914 , whose input proteome coverage (\%) was \% 0.16 and matched with pathogenic tree families (Table 9).

\section{Phylogenetic analysis}

Phylogenetic analysis constructed by inserting genome into species tree version 2.2.0 through Kbase platform. The phylogenetic analysis revealed that the multidrug-resistant Campylobacter fetus subspecies, which were isolated from sheath wash and obtained from bulls that were from the North-West province in South Africa, were highly similar and clustered tightly into one specific phylogenetic subgroups in the phylogenetic tree as it has been presented in figure 10 .

Table 4. Kruskal Wallis test for association between the area in which the samples were collected and the occurrence of Campylobacter fetus subspecies

\begin{tabular}{lcc}
\hline Bacteria & & Resistance \\
\hline Campylobacter fetus subsp. fetus & Chi-Square & 34.652 \\
& $\mathrm{df}$ & 13 \\
& $\mathrm{p}$-value & 0.001 \\
\hline & Chi-Square & 19.705 \\
Campylobacter fetus subsp. venerealis & $\mathrm{df}$ & 13 \\
& p-value & 0.103 \\
\hline
\end{tabular}

a. Kruskal Wallis Test; b. Grouping Variable: Antibiotic; df: the degree of freedom

Table 5 Association between the area in which samples were collected and the occurrence of Campylobacter fetus subspecies.

\begin{tabular}{llcc}
\hline Chi-Square Tests & Value & df & p-value \\
\hline Pearson Chi-Square & 0.728 & 3 & 0.867 \\
\hline
\end{tabular}


Table 6. Relationship between Campylobacter fetus subspecies resistance and different antibiotics tested against

\begin{tabular}{|c|c|c|}
\hline Antibiotic & Statistical test & Resistance \\
\hline \multirow{2}{*}{ Tetracycline } & Mann-Whitney U & 361.500 \\
\hline & $\mathrm{P}$-value & 0.287 \\
\hline \multirow{2}{*}{ Doxycycline } & Mann-Whitney U & 384.000 \\
\hline & $\mathrm{P}$-value & 0.552 \\
\hline \multirow{2}{*}{ Ampicillin } & Mann-Whitney U & 413.500 \\
\hline & $\mathrm{P}$-value & 0.926 \\
\hline \multirow{2}{*}{ Amoxicillin } & Mann-Whitney U & 400.000 \\
\hline & P-value & 0.744 \\
\hline \multirow{2}{*}{ Erythromycin } & Mann-Whitney U & 378.500 \\
\hline & $\mathrm{P}$-value & 0.508 \\
\hline \multirow{2}{*}{ Azithromycin } & Mann-Whitney U & 361.500 \\
\hline & $\mathrm{P}$-value & 0.475 \\
\hline \multirow{2}{*}{ Neomycin } & Mann-Whitney U & 348.000 \\
\hline & $\mathrm{P}$-value & 0.308 \\
\hline \multirow{2}{*}{ Streptomycin } & Mann-Whitney U & 328.500 \\
\hline & P-value & 0.108 \\
\hline \multirow{2}{*}{ Gentamicin } & Mann-Whitney U & 390.500 \\
\hline & $\mathrm{P}$-value & 0.638 \\
\hline \multirow{2}{*}{ Ciprofloxacin } & Mann-Whitney U & 356.000 \\
\hline & P-value & 0.275 \\
\hline \multirow{2}{*}{ Nalidixic acid } & Mann-Whitney U & $314.500^{*}$ \\
\hline & $\mathrm{P}$-value & 0.039 \\
\hline \multirow{2}{*}{ Norfloxacin } & Mann-Whitney U & 356.000 \\
\hline & $\mathrm{P}$-value & 0.300 \\
\hline \multirow{2}{*}{ Enrofloxacin } & Mann-Whitney U & 410.000 \\
\hline & P-value & .887 \\
\hline \multirow{2}{*}{ Chloramphenicol } & Mann-Whitney U & 376.000 \\
\hline & P-value & 0.448 \\
\hline
\end{tabular}

a. Grouping Variable: Bacteria

Table 7. Genes resistant encoded in Campylobacter fetus subsp. venerealis NW_ME2 and Campylobacter fetus subsp fetus NW_ME1

\begin{tabular}{|c|c|c|}
\hline Genes & Name & Function \\
\hline YkkCD & Broad-specificity multidrug efflux pump $Y k k C$ & EFP-CAR \\
\hline YKKCD & Broad-specificity multidrug efflux pump $Y k k C D$ & EFP-CAR \\
\hline CmeABC & Multidrug efflux system, membrane fusion component $\Rightarrow$ CmeA & EFP-CAR \\
\hline $\mathrm{CmeABC}$ & Transcriptional repressor of $\mathrm{CmeABC}$ operon, $\mathrm{CmeR}$ & RME-ARGe \\
\hline $\mathrm{CmeABC}$ & Multidrug efflux system, outer membrane factor lipoprotein $\Rightarrow \mathrm{CmeC}$ & EFP-CAR \\
\hline CmeABC & Multidrug efflux system, inner membrane proton/drug antiporter (RND type) $\Rightarrow>C m e B$ & EFP-CAR \\
\hline gidB & 16S rRNA (guanine(527)-N(7))-methyltransferase & $\mathrm{Ge}-\mathrm{CR}-\mathrm{Ab}$ \\
\hline rpoB & DNA-directed RNA polymerase beta subunit & AT-TSsp \\
\hline rpoC & DNA-directed RNA polymerase beta' subunit & AT-TSsp \\
\hline rho & Transcription termination factor Rho & AT-TSsp \\
\hline Ddl & D-alanine ligase & AT-TSsp \\
\hline MacB & Macrolide export ATP-binding/permease protein MacB & EFP-CAR \\
\hline MacA & Macrolide-specific efflux protein MacA & EFP-CAR \\
\hline 7a-HSDH-like & Enoyl-[acyl-carrier-protein] reductase, 7 -alpha-HSDH-like $=>$ refractory to triclosan & AT-REPLp \\
\hline MurA & UDP-N-acetylglucosamine 1-carboxyvinyltransferase & AT-SUSCsp \\
\hline dxr & 1-deoxy-D-xylulose 5-phosphate reductoisomerase & AT-SUSCsp \\
\hline EF-G & Translation elongation factor $\mathrm{G}$ & AT-SUSCsp \\
\hline EF-Tu & Translation elongation factor $\mathrm{Tu}$ & AT-SUSCsp \\
\hline $\mathrm{NimB}$ & Nitroimidazole resistance protein $\mathrm{NimB}$ & A-Inc ENZ \\
\hline Iso-tRNA & Isoleucyl-tRNA synthetase & AT-SUSCsp \\
\hline S10p & SSU ribosomal protein $\mathrm{S} 10 \mathrm{p}(\mathrm{S} 20 \mathrm{e})$ & AT-SUSCsp \\
\hline inhA, fabI & Enoyl-[acyl-carrier-protein] reductase [NADH] & AT-SUSCsp \\
\hline Alr & Alanine racemase & AT-SUSCsp \\
\hline PgsA & CDP-diacylglycerol--glycerol-3-phosphate 3-phosphatidyltransferase & Prot alt CWCh-CAR \\
\hline gyrB & DNA gyrase subunit B & AT-SUSCsp \\
\hline gyrA & DNA gyrase subunit A & AT-SUSCsp \\
\hline $\mathrm{S} 12 \mathrm{p}$ & SSU ribosomal protein $\mathrm{S} 12 \mathrm{p}$ & AT-SUSCsp \\
\hline TolC & Outer membrane protein TolC & Out Mem EF Prot-CAR \\
\hline
\end{tabular}

EFP-CAR (Efflux pump conferring antibiotic resistance), RME-ARGe (regulator modulating the expression of antibiotic resistance genes), Ge-CR$\mathrm{Ab}$ (Gene conferring resistance via absence), AT-TSsp (antibiotic target in susceptible species ), AT-REPLp (antibiotic target replacement protein), AT-SUSCsp (antibiotic target in susceptible species), A-Inc ENZ (antibiotic inactivation enzyme), Prot alt CWCh-CAR (protein-altering cell wall charge conferring antibiotic resistance), Out Mem EF Prot-CAR (Out membrane efflux protein conferring antibiotic resistance). 
Table 8. Virulence factors encoded in Campylobacter fetus subspecies Isolated from sheath wash

\begin{tabular}{|c|c|c|c|}
\hline Property & Genes & Product & Classification \\
\hline Virulence factor & fliI & Flagellum-specific ATP synthase FliI & $\begin{array}{l}\text { Motility, } \\
\text { Chemotaxis, } \\
\text { Invasion, Phase variation }\end{array}$ \\
\hline Virulence factor & CheY & $\begin{array}{l}\text { Chemotaxis regulator - transmits chemoreceptor signals to } \\
\text { flagellar motor components CheY }\end{array}$ & Chemotaxis, motility \\
\hline Virulence factor & GidA & $\begin{array}{l}\text { tRNA uridine 5-carboxymethylaminomethyl modification } \\
\text { enzyme GidA }\end{array}$ & Invasion \\
\hline Virulence factor & flgC & Flagellar basal-body rod protein FlgC & Motility, Chemotaxis, Invasion, Phase variation \\
\hline Virulence factor & pseB & UDP-N-acetylglucosamine 4,6-dehydratase (inverting) & $\begin{array}{l}\text { Biosynthesis, } \\
\text { synthesis of both functional flagella and } \\
\text { lipopolysaccharides }\end{array}$ \\
\hline Virulence factor & fliP & Flagellar biosynthesis protein FliP & Motility, Chemotaxis, Invasion, Phase variation \\
\hline Virulence factor & flhA & Flagellar biosynthesis protein FlhA & Motility, Chemotaxis, Invasion, Phase variation \\
\hline Virulence factor & fliM & Flagellar motor switch protein FliM & Motility \\
\hline Virulence factor & fliQ & Flagellar biosynthesis protein FliQ & Motility, Chemotaxis, Invasion, Phase variation \\
\hline Virulence factor & fliN & Flagellar motor switch protein FliN & Motility, Chemotaxis, Invasion, Phase variation \\
\hline Virulence factor & CdtA & Cytolethal distending toxin subunit A & Cytotoxin production \\
\hline Virulence factor & $\mathrm{CdtB}$ & Cytolethal distending toxin subunit B & Cytotoxin production \\
\hline Virulence factor & $\mathrm{CdtC}$ & Cytolethal distending toxin subunit $\mathrm{C}$ & Cytotoxin production \\
\hline Virulence factor & MotA & Flagellar motor rotation A & Motility \\
\hline Virulence factor & MotB & Flagellar motor rotation $\mathrm{B}$ & Motility \\
\hline Virulence factor & VirB3 & $\begin{array}{l}\text { Inner membrane protein forms channel for type IV secretion of } \\
\text { T-DNA complex }\end{array}$ & Adhesion and colonization \\
\hline Virulence factor & VirB4 & $\begin{array}{l}\text { ATPase required for both assembly of type IV secretion } \\
\text { complex and secretion of T-DNA complex }\end{array}$ & Adhesion and colonization \\
\hline Virulence factor & VirB10 & $\begin{array}{l}\text { Inner membrane protein of type IV secretion of T-DNA } \\
\text { complex, TonB-like }\end{array}$ & Adhesion and colonization \\
\hline Virulence factor & VirB8 & $\begin{array}{l}\text { Inner membrane protein forms channel for type IV secretion of } \\
\text { T-DNA complex }\end{array}$ & Adhesion and colonization \\
\hline Virulence factor & VirB9 & $\begin{array}{l}\text { Outer membrane and periplasm component of type IV } \\
\text { secretion of T-DNA complex has secretin-like domain }\end{array}$ & Adhesion and colonization \\
\hline Virulence factor & VirB9 & $\begin{array}{l}\text { Forms the bulk of type IV secretion complex that spans outer } \\
\text { membrane and periplasm }\end{array}$ & Adhesion and colonization \\
\hline Virulence factor & VirB5 & Minor pilin of type IV secretion complex & Adhesion and colonization \\
\hline Virulence factor & VirB1 & $\begin{array}{l}\text { Bores hole in peptidoglycan layer allowing type IV secretion } \\
\text { complex assembly }\end{array}$ & Adhesion and colonization \\
\hline Virulence factor & $\mathrm{CiaB}$ & Campylobacter invasion antigen B & Invasion, colonization \\
\hline Virulence factor & SLP & S-Layer protein & Adherence, evasion \\
\hline Virulence factor & VirB11 & $\begin{array}{l}\text { ATPase required for both assembly of type IV secretion } \\
\text { complex and secretion of T-DNA complex }\end{array}$ & Adhesion and Colonization \\
\hline Virulence factor & VirD4 & Type IV secretion system protein & Adhesion and colonization \\
\hline Virulence factor & VirD4 & Like coupling protein & Adhesion and colonization \\
\hline Virulence factor & fic & Fic domain protein, BT_4222 type & Adhesion and colonization \\
\hline Virulence factor & TrbE & Conjugative transfer protein TrbE & Adhesion and colonization \\
\hline Virulence factor & TrbD & Conjugative transfer protein TrbD & Adhesion and colinization \\
\hline Virulence factor & TrbC & Conjugative transfer protein TrbC & Adhesion and colonization \\
\hline Virulence factor & TrbI & Conjugative transfer protein TrbI & Adhesion and colonization \\
\hline Virulence factor & TrbG & Conjugative transfer protein TrbG & Adhesion and colonization \\
\hline Virulence factor & TrbF & Conjugative transfer protein $\mathrm{TrbF}$ & Adhesion and colonization \\
\hline Virulence factor & TrbL & Conjugative transfer protein $\mathrm{TrbL}$ & Adhesion and colonization \\
\hline Virulence factor & TrbJ & Conjugative transfer protein TrbJ & Adhesion and colonization \\
\hline Virulence factor & TraT & $\begin{array}{l}\text { IncF plasmid conjugative transfer surface exclusion protein } \\
\text { TraT }\end{array}$ & Adhesion and colonization \\
\hline Virulence factor & TrbB & Conjugative transfer protein TrbB & Adhesion and colonization \\
\hline
\end{tabular}

Table 9. Matched pathogenic families with Campylobacter fetus subsp. fetus NW_ME1

\begin{tabular}{|c|c|c|c|c|c|}
\hline Accession No & Organism & Class & Protein function & Protein number & Identity (\%) \\
\hline CP000792 & $\begin{array}{l}\text { Campylobacter. } \\
\text { concisus } 13826 \text { complete } \\
\text { genome }\end{array}$ & Epsilonproteo bacteria & $\begin{array}{l}\text { Thymidylate synthase, } \\
\text { flavin dependent }\end{array}$ & EAT99108 & 80.1 \\
\hline CP000767 & $\begin{array}{l}\text { Campylobacter. curvus } \\
525.92 \text { Complete genome }\end{array}$ & Epsilonproteo bacteria & Ribosomal protein S2 & EAU00943 & 81.25 \\
\hline CP000538 & $\begin{array}{l}\text { Campylobacter. Jejune } \\
\text { subsp. jejune } 81-176 \\
\text { Complete genome }\end{array}$ & $\begin{array}{l}\text { Epsilonproteo } \\
\text { bacteria }\end{array}$ & Ribosomal protein L22 & EAQ72756 & 85.45 \\
\hline
\end{tabular}


CmeB

Figure 2. Campylobacter fetus subsp. venerealis: RND efflux system inner membrane transporter CmeB, Location: 2,897-2,960 (+ Strand). DNA Lengh 63

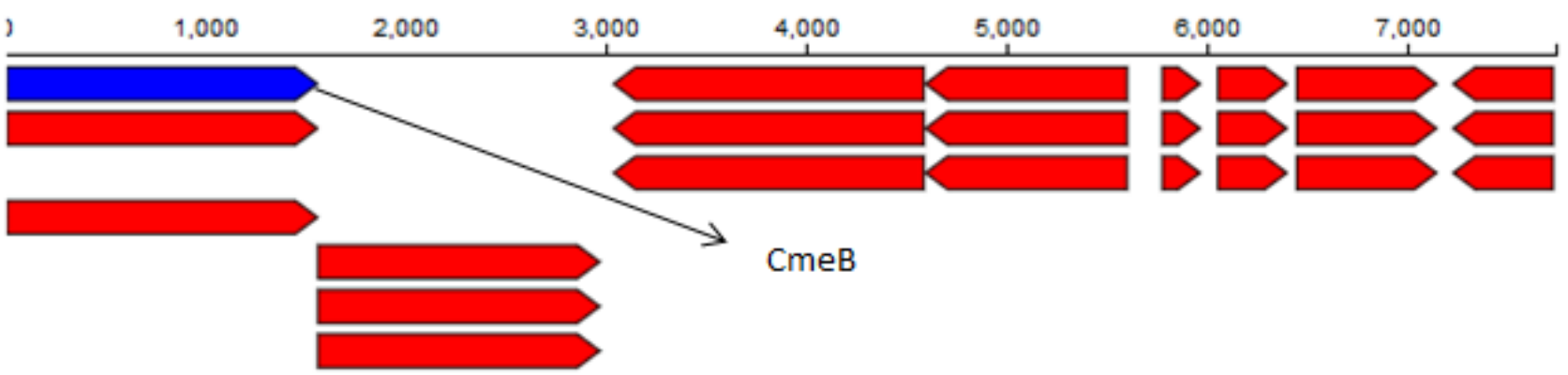

Figure 3. Campylobacter fetus subsp. fetus: RND efflux inner membrane transporter CmeB, Location: 2-1,562 (+ Strand). DNA Length 1560

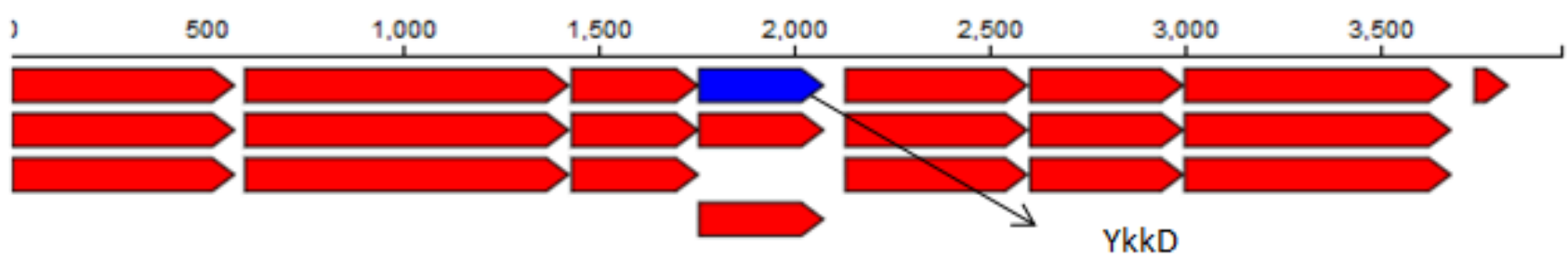

Figure 4. Campylobacter fetus subsp. fetus: Broad-specificity multidrug efflux pumps YkkD. Location 1, 757 - 2, 078 (+ Strand), DNA Length: 321

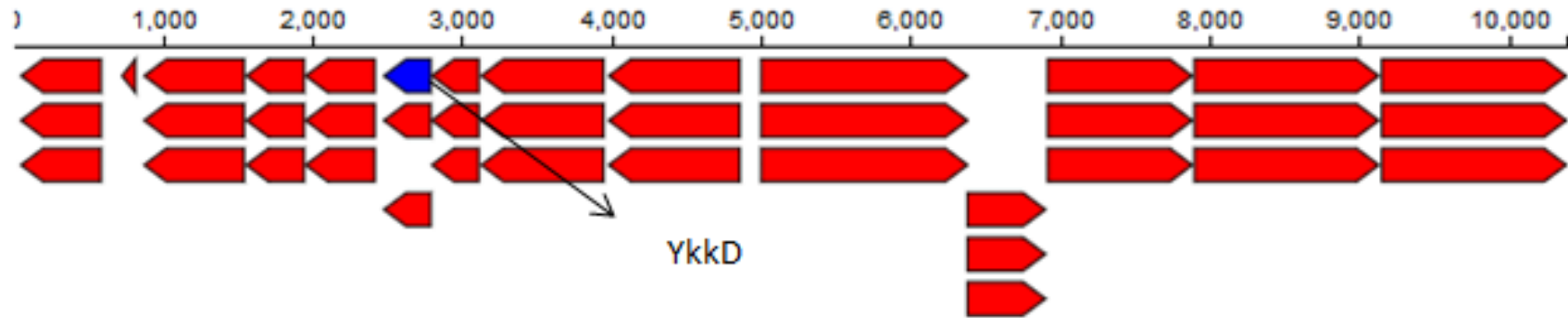

Figure 5. Campylobacter fetus subsp. venerealis: Broad-specificity multidrug efflux pumps YkkD. Location: 2, $471-2$, 792 (- Strand), DNA Length: 321

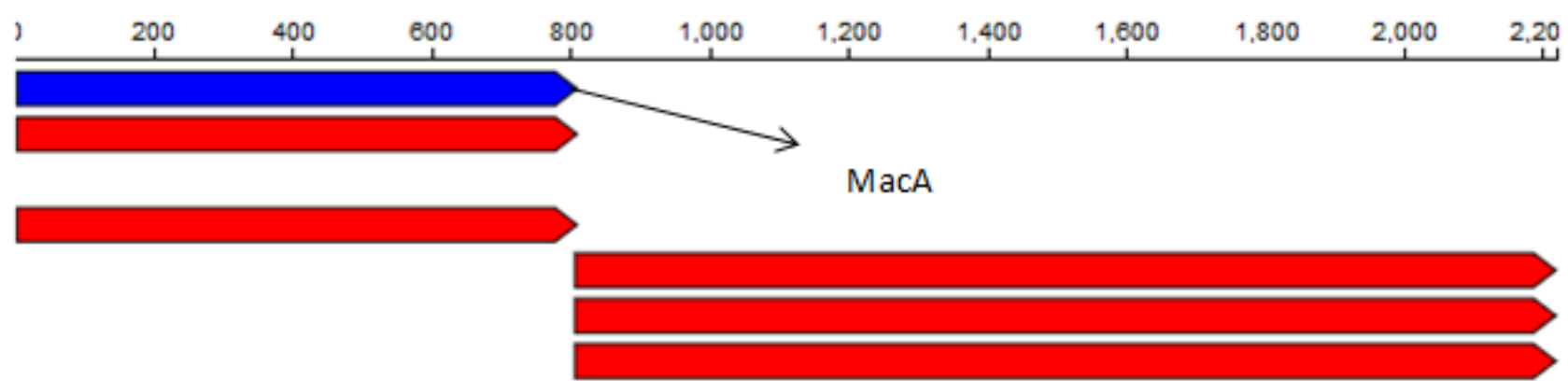

Figure 6. Campylobacter fetus subsp. fetus: Macrolide efflux protein MacA. Location: 2 - 809 (+ Strand), DNA Length: 807 
MacA

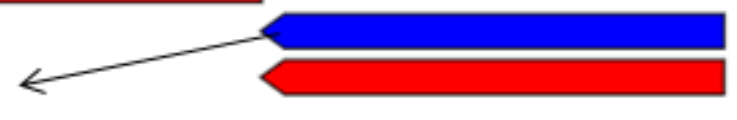

Figure 7. Campylobacter fetus subsp. venerealis: Macrolide specific efflux protein MacA. Location: 2, 599 - 3, 784 (Strand), DNA Length: 1, 185

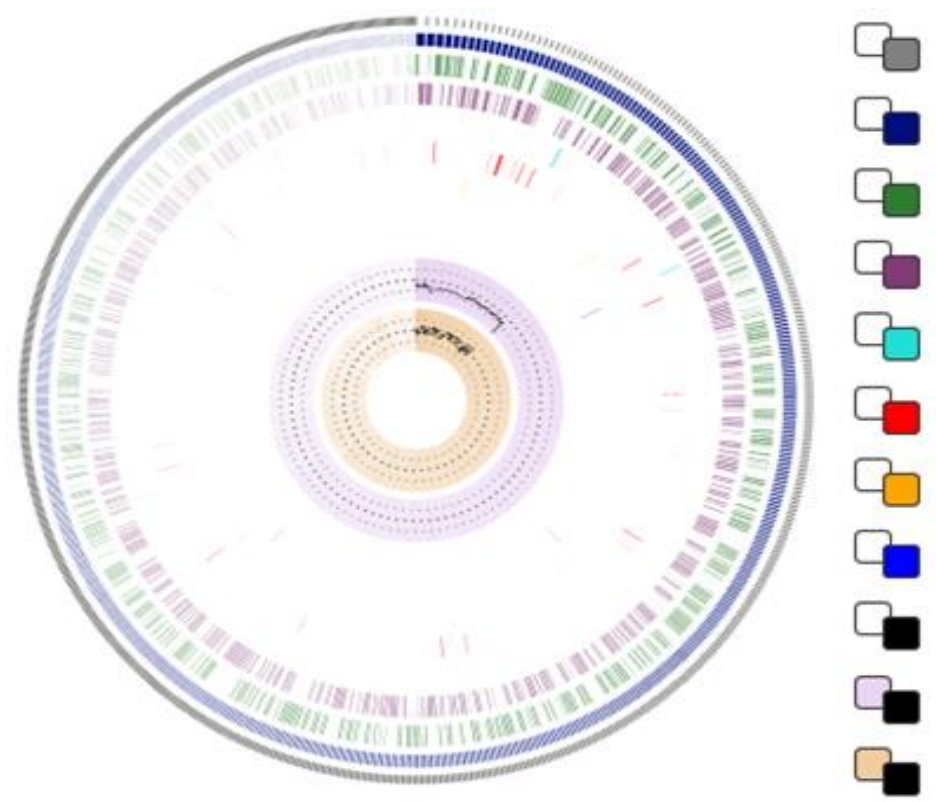

Position Label (Mbp)

Contigs/Chromosomes

CDS - FWD

CDS - REV

Non-CDS Features

AMR Genes

VF Genes

Transporters

Drug Targets

GC Content

GC Skew

Figure 8. Circular genome map of Campylobacter. fetus subsp. fetus NW_ME1. The overview of the genome map of Campylobacter fetus subsp. fetus NW_ME1 generated via Patrick annotation version 3.6.3. The tracks on the viewer are displayed as concentric rings, from outermost to innermost: Position, Contigs, CDS-Forwards, CDS- Reverse, Non-CDS features, GC content, and GC skew

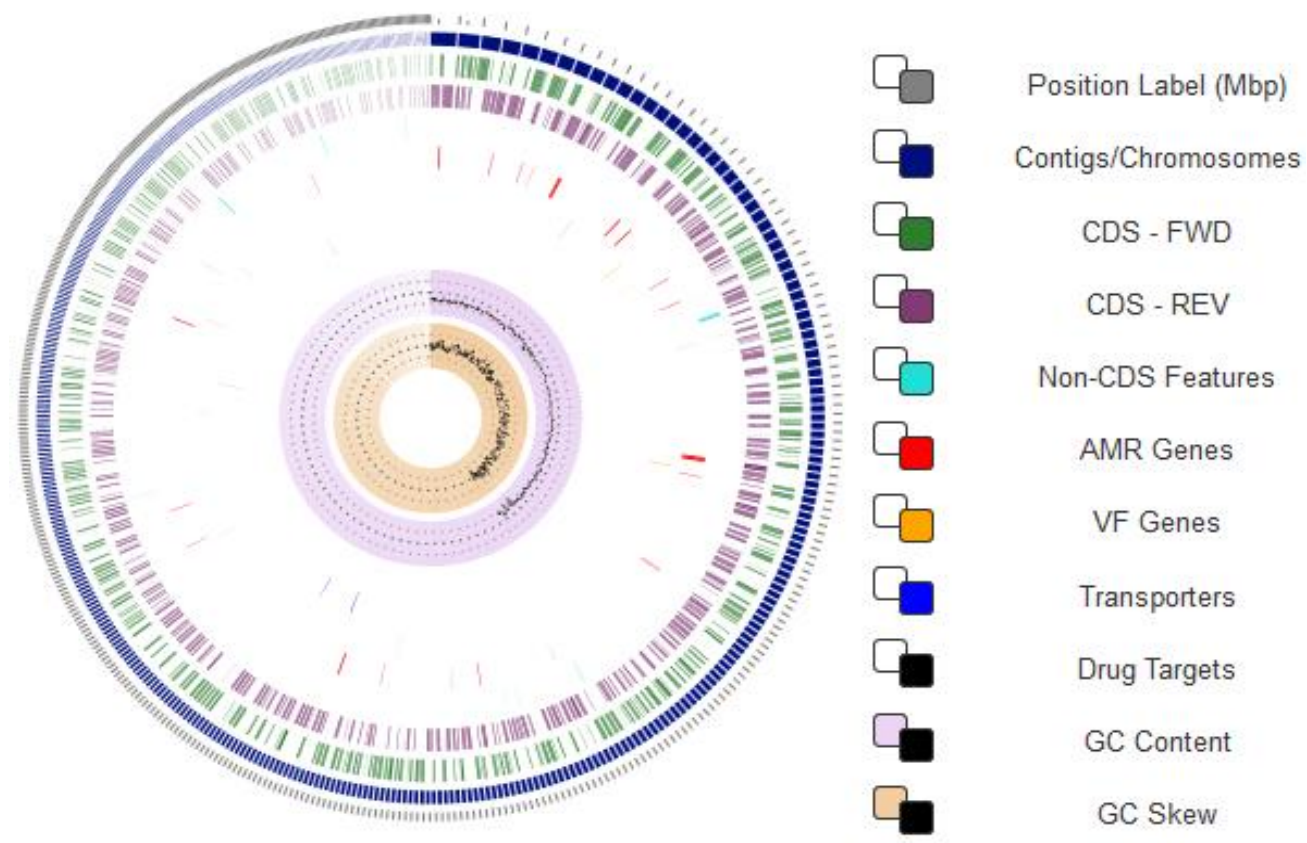

Figure 9. Circular genome map of Campylobacter fetus subsp. venerealis NW_ME2 generated using Patrick annotationcircular, from outside to inner ring contigs, CDS on the forward, CDS on the reverse, non CDS features, CDS with homology to known antimicrobial resistance genes, virulence genes, transports, drug targets GC content and GC skew. 


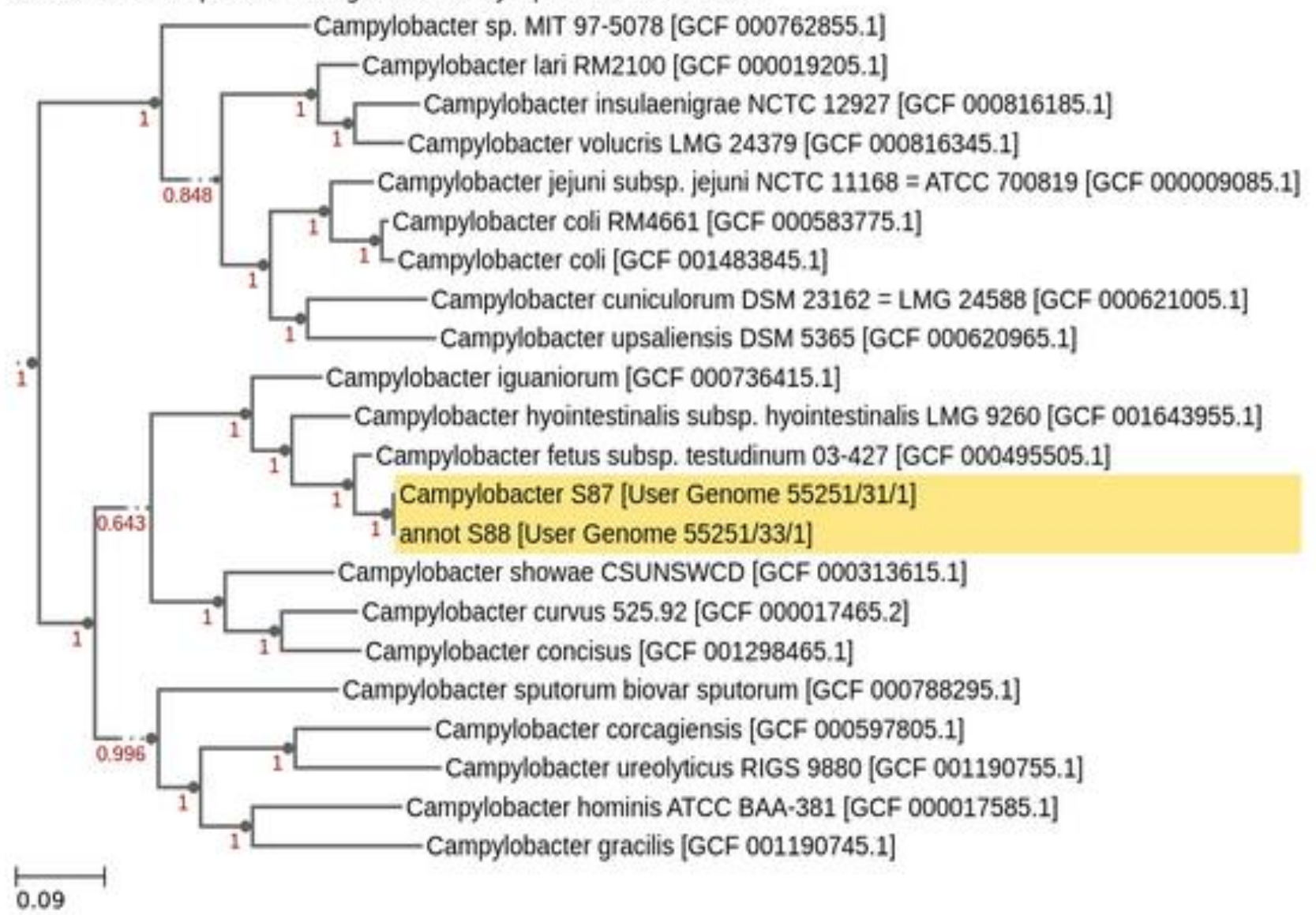

Figure 10. Whole-genome Neighbor joining phylogenetic tree. Phylogenetic tree of the complete genome of Campylobacter fetus subsp. fetus NW_ME1 and venerealis NW_ME2 align with 20 complete Campylobacter genomes; both Campylobacter fetus subspecies isolated in this study expressedmore significant homology. The digits indicated the distance or divergence between species (genome) used in the tree. The scale bar indicates 0.09 nucleotide substitutions per nucleotide position

\section{DISCUSSION}

The current study revealed that most of the Campylobacter fetus subspecies were multidrug-resistant to almost all the antibiotics tested against, as demonstrated in tables 2 and 3. The resistance of Campylobacter fetus subsp. fetus to different antibiotics was also reported in a study on the temporal prevalence of antimicrobial resistance in Campylobacter spp. from beef cattle in Alberta Feedlots in Canada. The researchers indicated that the Campylobacter fetus (39\%) was resistant to doxycycline and tetracycline. The same study presented that isolated Campylobacter fetus (97\%) were highly resistant to nalidixic acid (Tremblay et al., 2003). This corroborates with the findings of this study, that $85.2 \%$ of Campylobacter fetus subsp. fetus and $61.3 \%$ of Campylobacter fetus subsp. venerealis were observed to be highly resistant against nalidixic acid. The resistance of Campylobacter fetus subsp. fetus was also reported by Kenar and Gökçe (2019). The bacteria were isolated from the livers of slaughtered ruminants and aborted ovine fetuses. Their results, based on the disk diffusion method, revealed that the highest resistance was manifested against ciprofloxacin and tetracycline $(45.45 \%)$ while the highest sensitivity was against gentamicin $(90.91 \%)$ and chloramphenicol $(100 \%)$ (Kenar and Gökçe, 2019).

These findings slightly differ from the results of the present study; though both studies used disc diffusion methods, it was observed that the resistance to ciprofloxacin and tetracycline was higher when compared with the previous study, respectively $63.0 \%$ and $59.3 \%$. On the other hand, the susceptibility of gentamicin and chloramphenicol was very low, $29.6 \%$, and $11.1 \%$, respectively. Tremblay and Gaudreau (1998) evaluated the susceptibility profile for a total number of 59 Campylobacter fetus subsp. fetus against eight antimicrobial agents using the disc diffusion method, agar dilution, and E-test. The findings of their study concluded that Campylobacter fetus subsp. fetus was observed to be susceptible to meropenem, gentamicin, ampicillin, and imipenem. Further, the findings revealed that Campylobacter fetus subsp. fetus (27\%) exhibited resistance to profile to tetracycline for both methods used disk diffusion and agar dilution (Tremblay and Gaudreau, 1998). 
The results of the current study were different from previous ones as isolated Campylobacter fetus were not all susceptible to ampicillin and gentamicin; furthermore, imipenem and meropenem were not used in this study; this difference might be related to the source of isolated bacteria. In the present study, the isolated bacteria were obtained from sheath wash, while the previous study was obtained from clinical samples. In this study, it was also observed the resistance of Campylobacter fetus subsp. venerealis to different antibiotics such as tetracycline, ampicillin, neomycin, streptomycin and other antimicrobial agents as presented in table 3.

The phenotypic antimicrobial resistance profile of Campylobacter fetus subsp. fetus and Campylobacter fetus subsp. venerealis have never yet been investigated and reported in South Africa; this was one of the primary reasons for this study to investigate the genes involved in the mechanism of resistance in these two subspecies. These results aligned with observation revealed by researchers in the past few decades. An increasing number of isolated Campylobacter spp. have developed resistance to fluoroquinolones and other antimicrobials such as macrolides, aminoglycosides, and betalactams. In the current study, resistance against these classes of antibiotics was observed among the isolated bacteria. There is strong evidence indicating that the spread and dissemination of antibiotic resistance observed in many organisms are linked to the use of the antimicrobial agent in animal production and veterinary medicine for growth promoter and diseases prevention, which is also observed in Campylobacter spp. in humans (Wieczorek and Osek, 2013). In this study, both Campylobacter fetus subsp. fetus and venerealis expressed resistance to nalidixic acid, which confirmed that resistance to nalidixic acid had been proved to be an important marker to distinguish between $C$. jejuni and C. fetus (Taylor et al., 1985).

The WGS analysis revealed that isolated Campylobacter fetus subsp. fetus and venerealis harbored 28 resistance genes and 39 virulence factors. Among the encoded resistance genes, some have never been reported in Campylobacter fetus subsp. fetus and venerealis, such as gidB, ykkc, ykkcd, rho, MacB, and MacA, suggesting a new upcoming mechanism of resistance in these subspecies. Among the genes encoded, some are genetic determinants for fluoroquinolone (gyrA and gryB), for chloramphenicol, and tetracycline (ykkc and ykkcd), for aminoglycoside (gidB). Additionally, multidrug resistance efflux pumps (CmeABC), Broad-specificity multidrug efflux pump (ykkc and ykkcd), efflux pump conferring antibiotic resistance (MacA and $\mathrm{MacB}$ ), and protein-altering cell wall charge conferring antibiotic resistance (PgsA) were among important CDS encoded. In Escherichia coli the expression of ykkc or ykkd was observing to induce phenotypic multidrug-resistant profile in bacteria, including resistance to neutral drugs, cationic and anionic, as it is shown in figure 4 (Jack et al., 2000). Further, the overexpression of these efflux pumps contributes to reducing susceptibility by decreasing the intracellular concentration of the antimicrobial agents (Nikaido and Pagès, 2012). This finding corroborates with the result of the current study, which observed the expression of different efflux pumps as they have been presented in table 7. The study revealed that collaborating MacB, in concert with the adaptor MacA and the outer membrane exit duct, TolC, could underpin the efflux of various drugs and export of virulence factors from multiple Gram-negative bacterial species. Additionally, a study revealed that in Gram-negative bacteria such as Stenotrophomonas maltophilia, Escherichia coli, and Salmonella spp MacA, MacB together with Tolc conferred resistance to numerous classes of antibiotics respectively aminoglycosides, macrolides as well as polymyxins (Nishi et al., 2003; Lin et al., 2014). These findings correlated with the results of the current study because MacA, MacB, and Tolc were encoded in both Campylobacter fetus subsp. fetus and venerealis, and this clarified why our isolated bacteria presented a resistance profile to macrolides and aminoglycosides antibiotics. On the other hand, in this study, the multidrug resistance efflux pumps $(\mathrm{Cme} A B C)$ were encoded in both isolated bacteria. The efflux pump (CmeABC) has been pointed out to have an important role in the emergence of fluoroquinolone-resistant Campylobacter (Yan et al., 2006). It has also been reported that CmeABC is an important multidrug efflux pump in Campylobacter species and contributes to its intrinsic and acquired resistance to a broad spectrum of antibiotics, including fluoroquinolone resistance (Lin et al., 2002; Pumbwe and Piddock, 2002; Lin et al., 2003). The CmeABC is distributed in tripartite multidrug efflux pumps, which consist of $\mathrm{CmeC}$ (outer membrane protein), CmeA (periplasmic fusion protein) and $\mathrm{CmeB}$ (inner membrane efflux transporter) figure 3 (Lin et al., 2002).

This finding aligned with the results of the present study, that different operon of the efflux pump were detected in Campylobacter fetus subsp. venerealis and Campylobacter fetus subsp. fetus. It has also been reported that these three proteins $(\mathrm{CmeA}),(\mathrm{CmeB})$ and $(\mathrm{CmeC})$ are encoded by a three-gene operon (cmeABC) and function together to form a membrane channel that extruded toxic substrates directly out of Campylobacter cells (Lin et al., 2002). The CmeABC contributed actively to the intrinsic and acquired resistance of Campylobacter species to structurally diverse antimicrobials (Lin et al., 2002; Pumbwe and Piddock, 2002; Luo et al., 2003).

Additionally, this multidrug efflux pump (CmeABC) is well known to confer resistance against bile. It is required for the growth of Campylobacter spp. in media containing bile that serves to colonize the intestinal tract in animals (Lin et al., 2003). Furthermore, In this study, the transcriptional repressor of CmeABC, operon CmeR was also detected in isolated bacteria subjected to the whole genome analysis. It has been revealed that $C m e R$ interacts with the cmeABC promoter and modulated the expression of cmeABC. On the other hand, the CmeR is known to improve the production and expression of a multidrug efflux pump (Lin et al., 2005). In the present study, the isolated bacteria were 
phenotypically resistant to macrolide tested against erythromycin and azithromycin. The genome sequencing reveals the presence of MacB (macrolide export ATP binding permease protein) as well as MacA gene as it has been shown in figures 6 and 7 .

It has been reported that $\mathrm{MacB}$, along with its periplasmic adaptor protein MacA, was first identified in Escherichia coli transporter genes as providing resistance to macrolide drugs in a strain lacking the major RND efflux pump AcrAB (Kobayashi et al., 2001). It is also known that these genes MacA and MacB have never been reported in Campylobacter fetus subspecies around Sound Africa, and this might be the first report. Another study investigated the role of MacAB in Gram-negative species such as Stenotrophomonas maltophilia and found that MacA and B together with Tolc conferred resistance to a variety of macrolides, aminoglycosides, and polymyxins in Gram-negative species (Nishi et al., 2003; Lin et al., 2014).

These findings corroborated with the results of this study, in which MacA and B were detected in isolated bacteria, and phenotypic resistance to macrolide and aminoglycoside was also observed. The auxiliary studies of the MacAB-Tolc framework examined by the Zgurskaya research facility illustrated that the ATPase movement of reconstituted MacB is subordinate to intaglio MacA. Assist, the same consider affirmed that MacAB interceded antimicrobial resistance in vivo requires the nearness of the external layer efflux channel TolC (Tikhonova et al., 2007). Tikhonova and his co-authors corroborated with the findings of this study that the presence of outer membrane efflux channel Tolc was detected in the isolated bacteria and observed in this study that the presence of gene gidB encoded with methyltransferase. This gene has been pointed out as conferring high-level antimicrobial resistance in Gram-negative such as salmonella (Mikheil et al., 2012). The research found out that the cancellation of gidB conferred resistance to the aminoglycoside antimicrobials, such as neomycin and streptomycin, whereas gidB protein had a noteworthy part within the modification of antimicrobial susceptibility (Mikheil et al., 2012). This might be the only explanation related to the aminoglycoside observed in this study. Moreover, alteration inside gidB has been linked to a low level of streptomycin resistance in some bacterial species and tall recurrence of the development of streptomycin-resistant mutants, which was too detailed in Campylobacter upsaliensis (Nishimura et al., 2007; Okamoto et al., 2007; Olkkola et al., 2015).

It has been moreover detailed that in microorganisms, gidB influenced antimicrobial sensitivity by a process that includes a post-transcriptional alteration (Mikheil et al., 2012). In this study, Broad-specificity multidrug efflux pump $Y k k C D$ was encoded in isolated bacteria; the gene was reported in Gram-positive Bacillus subtilis and Escherichia coli. It has been reported that in Escherichia coli strain DH5 $\alpha$ when the gene ykkc and ykkd were expressed, a broad-spectrum multidrug resistance phenotype was observed to different antimicrobial agents, such as chloramphenicol, streptomycin, and tetracycline. Further resistance was also observed to a broader range of toxic compounds (Jack et al., 2000). Considering different genes encoded in our isolated bacteria subjected to the WGS, it is possible to conclude that the multidrug resistance observed in this study might be linked to these genes.

Moreover, some genes found in this study, such as gibB, ykkc, ykkcd, MacA, and MacB, have never been reported in Campylobacter fetus subsp. fetus and Campylobacter fetus subsp. venerealis isolated from the sheath wash, their presence in this species might be considered as a new upcoming mechanism of resistance in Campylobacter fetus subspecies.

The WGS analysis also revealed presence of numerous genes (table 8) associated with bacterial motility and chemotaxis (fliI, flip, flhA, fliM, cheY, fliQ and fliN ). Among them, FliM, FliG, and FliN are important proteins which form switch complex $\mathrm{C}$ ring. This complex interacts with the CheY and CheZ chemotaxis proteins, in addition to contact components of the motor that determine the direction of flagellar rotation. In the current study, flagellar motor rotation (MotA and B) were also detected. It has been reported that these flagella conferred swimming motility and distinctive darting motility, which was necessary for infection of humans to cause diarrheal disease and in animals to promote commensalism (Balaban and Hendrixson, 2011). The FliM and FliN proteins have also been reported in Escherichia coli and Salmonella spp, where they form complexes beneath FliG (Paul et al., 2011; McDowell et al., 2016). The middle domain of FliM contains a CheC-like domain that was not detected in the present study, which forms a continuous belt in the middle of the $\mathrm{C}$ ring (Park et al., 2006; Sircar et al., 2015).

In contrast, CheY, together with FliM impact the clockwise or counter-wise motor rotation for chemotaxis (Mathews et al., 1998; Szurmant et al., 2003; Ahn et al., 2013). It has also been reported that in bacterial FliG, FliM, and FliN proteins act as a molecular switch, enabling the motor to spin in both counterclockwise and clockwise directions (Morimoto and Minamino, 2014) while MotA and MotB act as a proton pathway to couple proton flow through the channel with torque generation (Blair and Berg, 1990). Another study reported that MotA/B complex of alkaliphilic Bacillus clausii could conduct both $\mathrm{H}^{+}$and $\mathrm{Na}^{+}$at different $\mathrm{pH}$ ranges (Terahara et al., 2008). Additionally study conducted on utilitarian bi-directional flagellar motor uncovered that the two rotor proteins, FliM and FliN, illustrated significantly enthusiastic turnovers between the motor and their cytoplasmic pools (Morimoto and Minamino, 2014). The FliM turnover played an important role in switching the direction of flagellar motor rotation (Delalez et al., 2010; Yuan et al., 2012). In the present study, the responsible gene for adhesion and colonization was identified as virB11. This gene has been detected in retail chicken breast and fecal samples obtained from chicken, suggesting that chicken is a 
potential factor that could invade human intestinal cells with the gene (Thakur et al., 2010; Lapierre et al., 2016). However, in this study, virB11 was detected from sheath wash obtained from the bull, which also suggested that cattle, in general, might constitute a risk factor in the transmission of these genes to humans. Present findings revealed that flagellar and adhesion genes were exceptionally preserved in Campylobacter species as suggested in other studies (Thakur et al., 2010; Koolman et al., 2015; Lapierre et al., 2016). In the current study, other virulence marker determinants, including Cytolethal Distending Toxin (A, B, and C), were present in the isolated bacteria. Other studies revealed that three subunits are required for full toxin activity (No et al., 2002; Lapierre et al., 2016). It has also been indicated that these genes played an important role in the host mucosal inflammatory response for Interleukin-8 (IL-8) released by intestinal cells (Perera et al., 2007). Moreover, these genes have been pointed to cause diarrhea by interfering with the division and differentiation of the intestinal crypt cells (Wieczorek et al., 2018). The ciaB marker, which plays a significant role both in the invasiveness and in the colonization of the epithelial cells, was also identified in Campylobacter fetus in this study. Moreover, this gene has been detailed to easier the conquering of epithelial cells of the gastrointestinal tract, where it delivered expanding harm to the columnar epithelial cells, which led to swelling and adjusting of attacked cells as a result of the cytotoxin and enterotoxin activities (Sopwith et al., 2006; Perera et al., 2007).

In this study, the type IV secretion system VirD4 was detected only in Campylobacterfetus subsp.fetus NW_ME1. The type IV translocation pilus is encoded by the gene VirB operon that consists of eleven genes (virB1-virB11); translocation is also dependent on an additional gene virD4, which encodes the type IV coupling protein T4CP (van der Graaf-van Bloois et al., 2016). These findings align with the results of the present study that virB5, virB1, virB9, virB8, virB4, virB3, and virD4 (like coupling protein) were detected in both isolated bacteria. In this study, fic domain protein was found in the isolated bacteria, and it has been reported that this gene had significant roles in multiple cellular processes, including disrupting the host cell processes that were important to pathogen survival and replication after transmission into eukaryotic cells (Worby et al., 2009). Furthermore, the surface layer protein was encoded in this study in both isolated bacteria, and it is well known that surface layer proteins are responsible for the pathogenicity of Campylobacter fetus subspecies (Blaser et al., 1987; Pei and Blaser, 1990). On the other hands, the study revealed that both the surface layer proteins and T4SS regions are suggested to have a role in the pathogenicity of Campylobacter fetus subspecies, and it might be possible that these features have a synergistic role in immune escape (van der Graafvan Bloois et al., 2016). Among the important genes encoded in this study, it was also observed the presence of GidA tRNA uridine 5-carboxymethyl aminomethyl modification enzyme, which is presented in table 8 . This gene has been considered to be part of the major virulence mechanism in Gram-positive and negative bacteria such as Escherichia coli, Salmonella spp and staphylococcus but never been reported in Campylobacter fetus subspecies (Shippy and Fadl, 2014).

\section{CONCLUSION}

The findings of this study indicated that Campylobacter fetus subsp. fetus and Campylobacter fetus subsp. venerealis subjected to antimicrobial tests were observed to be multidrug resistance to almost antibiotics tested against. The WGS analysis of Campylobacter fetus subsp. fetus NW_ME1 and Campylobacter fetus subsp. venerealis NW_ME2 revealed the presence of different genes involved in the mechanism of resistance of the isolated bacteria and virulence factors that are susceptible to initiate the disease. Furthermore, the study encoded genes that have never been reported in Campylobacter fetus subsp. fetus and venerealis such as Broad-specificity multidrug efflux pump (ykkc and ykkd), 16S rRNA (guanine ${ }^{527}-\mathrm{N}^{7}$ ) methyltransferase (gidB), efflux pump conferring antibiotic resistance (MacA and MacB) suggesting a new resistance mechanism among these subspecies.

\section{DECLARATIONS}

\section{Acknowledgments}

The author acknowledges the technical support and advice of various colleagues working in the diagnostic and molecular laboratory of the Animal Health Department.

\section{Authors' contributions}

Mulunda Mwanza provided the funds for the project, participated in the drafting of the research proposal, and participated in the revisions of the final draft of the manuscript. Lubanza Ngoma provided technical support in the laboratory. Mpinda Edoaurd Tshipamba, designed the project, elaborated its plan, collected the samples, conducted the laboratory work, analyzed the results, and wrote the final manuscript for the publication.

\section{Competing interests}

The authors of this study declared that there is no financial conflict related to this work, which can negatively impact its publication. 
Acke E, McGill K, Golden O, Jones B, Fanning, and Whyte P (2009). A comparison of different culture methods for the recovery of campylobacter species from pets. Zoonoses and public health, 56 (9-10): 490-495. DOI: https://doi.org/10.1111/j.18632378.2008.01205.x .

Ahn D-R, Song H, Kim J, Lee S, and Park SY (2013). The crystal structure of an activated thermotoga maritima chey with n-terminal region of flim. International journal of biological macromolecules, 54: 76-83. DOI: https://doi.org/10.1016/j.ijbiomac.2012.12.003 .

Altschul SF, Madden TL, Schäffer AA, Zhang J, Zhang Z, Miller W, and Lipman DJ (1997). Gapped blast and psi-blast: A new generation of protein database search programs. Nucleic acids research, 25 (17): 3389-3402. DOI: https://doi.org/10.1093/nar/25.17.3389

Arkin AP, Cottingham RW, Henry CS, Harris NL, Stevens RL, Maslov S, Dehal P, Ware D, Perez F, Canon S et al. (2018). Kbase: The united states department of energy systems biology knowledgebase. Nature biotechnology, 36 (7): 566. DOI: https://doi.org/ 10.1038/nbt.4163

Aziz RK, Bartels D, Best AA, DeJongh M, Disz T, Edwards RA, Formsma K, Gerdes S, Glass EM, Kubal M et al. (2008). The rast server: Rapid annotations using subsystems technology. BMC genomics, 9 (1): 75. DOI: https://doi.org/10.1186/1471-2164-9$\underline{75}$.

Balaban M, and Hendrixson DR (2011). Polar flagellar biosynthesis and a regulator of flagellar number influence spatial parameters of cell division in campylobacter jejuni. PLoS pathogens, 7 (12): e1002420. DOI: https://doi.org/10.1371/journal.ppat.1002420

Bankevich A, Nurk S, Antipov D, Gurevich AA, Dvorkin M, Max A, Pham S, Tesler G, Vyahhi N, Teslere G, et al. (2012). Spades: A new genome assembly algorithm and its applications to single-cell sequencing. Journal of computational biology, 19 (5): $455-$ 477. DOI: https//doi.org/ $10.1089 / \mathrm{cmb} .2012 .0021$.

Bellows DS, Ott SL, and Bellows RA (2002). Cost of reproductive diseases and conditions in cattle. The Professional Animal Scientist, 18 (1): 26-32. DOI: https://doi.org/10.15232/S1080-7446(15)31480-7.

Blair DF, and Berg HC (1990). The mot A protein of e. Coli is a proton-conducting component of the flagellar motor. Cell, 60 (3): 439-449. DOI: https://doi.org/10.1016/0092-8674(90)90595-6

Blaser MJ, Smith PF, Hopkins JA, Heinzer I, Bryner JH, and Wang WL (1987). Pathogenesis of campylobacter fetus infections: Serum resistance associated with high-molecular-weight surface proteins. Journal of Infectious Diseases, 155(4): 696-706. DOI: https://doi.org/10.1093/infdis/155.4.696 .

Bolger AM, Lohse M and Usadel B (2014). Trimmomatic: A flexible trimmer for illumina sequence data. Bioinformatics, 30(15): 2114-2120. DOI: https://doi.org/10.1093/bioinformatics/btu170.

Delalez NJ, Wadhams GH, Rosser G, Xue Q, Brown MT, Dobbe LM, Berry RM, Leake MC, and Armitage JP (2010). Signaldependent turnover of the bacterial flagellar switch protein flim. Proceedings of the national academy of sciences, 107(25): 11347-11351. DOI: https://doi.org/10.1073/pnas.1000284107

Di Giannatale E, Di Serafino G, Zilli K, Alessiani A, Sacchini L, Sacchini L, Garofolo G, Aprea G, and Marotto F (2014). Characterization of antimicrobial resistance patterns and detection of virulence genes in campylobacter isolates in italy. Sensors, 14 (2): 3308-3322. DOI: https://doi.org/ 10.3390/s140203308.

Erickson NE, Lanigan E, Waugh T, Gesy K, and Waldner C (2017). Evaluation of long-acting oxytetracycline and a commercial monovalent vaccine for the control of campylobacter fetus subsp. Venerealis infection in beef bulls. The Canadian Veterinary Journal, 58(10): 1051. PMID: 28966354.

Guerra AG, Chaban B, Hill JE, Waldner CL, and Hendrick SH (2014). Clinical sensitivity and specificity of a real-time pcr assay for campylobacter fetus subsp venerealis in preputial samples from bulls. American journal of veterinary research, 75 (9): $851-860$.

DOI: https://doi.org/10.2460/ajvr.75.9.851 .

Haft DH, DiCuccio M, Badretdin A, Brover V, Chetvernin V, O'Neill K, Li W, Chitsaz F, Derbyshire M, Gonzalez et al. (2018). Refseq: An update on prokaryotic genome annotation and curation. Nucleic acids research, 46 (D1): D851-D860. DOI:https://doi.org/10.1093/nar/gkx1068

institute Cals (2013). Performance standards for antimicrobial susceptibility testing; twenty-third informational supplement. Secondary title. Twenty-Third Informational Supplement ed. Available at : www.clsi.org

Jack DL, Storms ML, Tchieu JH, Paulsen IT, and Saier MH (2000). A broad-specificity multidrug efflux pump requiring a pair of homologous smr-type proteins. Journal of bacteriology 182 (8): 2311-2313. DOI: https://doi.org/10.1128/jb.182.8.23112313.2000.

BAKWS JC and Turck M (1966). Antibiotic susceptibility testing by a standardized single disk method. Am J Clin Pathol. 45: $493-$ 496. PMID: 5908210.

KENAR B, and GÖKÇE M (2019). Antibiotic resistance to campylobacter spp. Isolated from the livers of slaughtered ruminants and aborted ovine fetuses. Kocatepe Veteriner Dergisi, 1-1. DOI: https://doi.org/10.30607/kvj.505685.

Kobayashi N, Nishino K, and Yamaguchi A (2001). Novel macrolide-specific abc-type efflux transporter in escherichia coli. Journal of bacteriology, 183(19): 5639-5644. DOI: https://doi.org/10.1128/JB.183.19.5639-5644.2001

Koolman L, Whyte P, Burgess C, and Bolton D (2015). Distribution of virulence-associated genes in a selection of campylobacter isolates. Foodborne pathogens and disease, 12(5): 424-432. DOI:https://doi.org/10.1089/fpd.2014.1883.

Lapierre L, Gatica MA, Riquelme V, Vergara C, Yañez JM, Martin BS, Saenz L, Vidal M, Martinez MC, Araya P et al (2016). Characterization of antimicrobial susceptibility and its association with virulence genes related to adherence, invasion, and cytotoxicity in campylobacter jejuni and campylobacter coli isolates from animals, meat, and humans. Microbial Drug Resistance, 22(5): 432-444. DOI: https://doi.org/10.1089/mdr.2015.0055. 
Lin J, Akiba M, Sahin O, and Zhang Q (2005). Cmer functions as a transcriptional repressor for the multidrug efflux pump cmeabc in campylobacter jejuni. Antimicrobial agents and chemotherapy, 49 (3): 1067-1075. DOI: https://doi.org/10.1128/AAC.49.3.1067-1075.2005 .

Lin J, Michel LO, and Zhang Q (2002). Cmeabc functions as a multidrug efflux system in campylobacter jejuni. Antimicrobial agents and chemotherapy, 46 (7): 2124-2131. DOI: https://doi.org/10.1128/aac.46.7.2124-2131.2002 .

Lin J, Sahin O, Michel LO and Zhang Q (2003). Critical role of multidrug efflux pump cmeabc in bile resistance and in vivo colonization of campylobacter jejuni. Infection and immunity, 71 (8): 4250-4259. DOI: https://doi.org/10.1128/IAI.71.8.4250$\underline{4259.2003}$.

Lin YT, Huang YW, Liou RS, Chang YC andYang TC (2014). Macabcsm, an abc-type tripartite efflux pump of stenotrophomonas maltophilia involved in drug resistance, oxidative and envelope stress tolerances and biofilm formation. Journal of Antimicrobial Chemotherapy, 69 (12): 3221-3226. DOI: https://doi.org/10.1093/jac/dku317 .

Luo N, Sahin O, Lin J, Michel LO, and Zhang Q (2003). In vivo selection of campylobacter isolates with high levels of fluoroquinolone resistance associated with gyra mutations and the function of the cmeabc efflux pump. Antimicrobial agents and chemotherapy, 47(1): 390-394. DOI: https://doi.org/10.1128/AAC.47.1.390-394.2003

Mak S, Xu Y, and Nodwell JR (2014). The expression of antibiotic resistance genes in antibiotic-producing bacteria. Molecular microbiology, 93(3): 391-402. DOI: https://doi.org/10.1111/mmi.12689.

Mathews MA, Tang HL and Blair DF (1998). Domain analysis of the flim protein of escherichia coli. Journal of bacteriology, 180 (21): 5580-5590. DOI: https://doi.org/10.1128/JB.180.21.5580-5590.1998.

McCool C, Townsend M, Wolfe S, Simpson M, Olm T, Jayawardhana GA, and Carney JV (1988). Prevalence of bovine veneral disease in the victoria river district of the northern territory: Likely economic effects and practicable control measures. Australian veterinary journal, 65 (5): 153-156. DOI:https://doi.org/ 10.1111/j.1751-0813.1988.tb14445.x .

McDowell MA, Marcoux J, McVicker G, Johnson S, Fong YH, Stevens R, Bowman LA, Degiacomi MT, Yan J, Wise A et al (2016). Characterisation of shigella spa33 and thermotoga flim $/ \mathrm{n}$ reveals a new model for c-ring assembly in t3ss. Molecular microbiology, 99 (4): 749-766. DOI: https://doi.org/ 10.1111/mmi.13267.

Mikheil DM, Shippy DC, Eakley NM, Okwumabua OE and Fadl AA (2012). Deletion of gene encoding methyltransferase (gidb) confers high-level antimicrobial resistance in salmonella. The Journal of antibiotics, 65 (4): 185-192. DOI:https://doi.org/ $10.1038 / \mathrm{ja}$.2012.5.

Morimoto YV, and Minamino T (2014). Structure and function of the bi-directional bacterial flagellar motor. Biomolecules, 4 (1): 217-234. DOI: https://doi.org/10.3390/biom4010217.

Morrell EL, Barbeito CG, Odeon C, Gimeno EJ, and Campero CM (2011). Histopathological, immunohistochemical, lectinhistochemical and molecular findings in spontaneous bovine abortions by campylobacter fetus. Reproduction in Domestic Animals, 46(2): 309-315. DOI: https://doi.org/10.1111/j.1439-0531.2010.01668.x.

Nikaido H, and Pagès JM (2012). Broad-specificity efflux pumps and their role in multidrug resistance of gram-negative bacteria. FEMS microbiology reviews, 36(2): 340-363. DOI: https://doi.org/10.1111/j.1574-6976.2011.00290.x .

Nishi J, Sheikh J, Mizuguchi K, Luisi B, Burland V, Boutin A, Rose DJ, Blattner FR, and Nataro JP (2003). The export of coat protein from enteroaggregative escherichia coli by a specific atp-binding cassette transporter system. Journal of Biological Chemistry, 278(46): 45680-45689. DOI: https://doi.org/10.1074/jbc.M306413200.

Nishimura K, Johansen SK, Inaoka T, Hosaka T, Tokuyama S, Tahara Y, Okamoto S, Kawamura F, Douthwaite S and Ochi K (2007). Identification of the rsmg methyltransferase target as 16s rrna nucleotide g527 and characterization of bacillus subtilis rsmg mutants. Journal of bacteriology, 189 (16): 6068-6073. DOI: https://doi.org/ 10.1128/JB.00558-07.

No HK, Park NY, Lee SH, and Meyers SP (2002). Antibacterial activity of chitosans and chitosan oligomers with different molecular weights. International journal of food microbiology, 74 (1-2): 65-72. DOI: https://doi.org/10.1016/s0168-1605(01)00717-6.

Okamoto S, Tamaru A, Nakajima C, Nishimura K, Tanaka, Tokuyama S, Suzuki Y, and Ochi K (2007). Loss of a conserved 7 methylguanosine modification in 16s rrna confers low-level streptomycin resistance in bacteria. Molecular microbiology, 63(4): 1096-1106. DOI: https://doi.org/ 10.1111/j.1365-2958.2006.05585.x

Olkkola S, Kovanen S, Roine J, Hänninen ML, Hielm-Björkman A, and Kvisto R (2015). Population genetics and antimicrobial susceptibility of canine campylobacter isolates collected before and after a raw feeding experiment. PloS one, 10(7): e0132660. DOI: https://doi.org/10.1371/journal.pone.0132660.

Park SY, Lowder B, Bilwes AM, Blair DF and Crane BR (2006). Structure of flim provides insight into assembly of the switch complex in the bacterial flagella motor. Proceedings of the national academy of sciences, 103 (32): 11886-11891. DOI:https://doi.org/ 10.1073/pnas.0602811103.

Paul K, Gonzalez-Bonet G, Bilwes AM, Crane BR and Blair D (2011). Architecture of the flagellar rotor. The EMBO journal, 30(14): 2962-2971. DOI:https://doi.org/ 10.1038/emboj.2011.188.

Pei Z and Blaser MJ (1990). Pathogenesis of campylobacter fetus infections. Role of surface array proteins in virulence in a mouse model. The Journal of clinical investigation 85 (4): 1036-1043. DOI:https://doi.org/ 10.1172/JCI114533 .

Perera VN, Nachamkin I, Ung H, Patterson JH, McConville MJ, Coloe PJ and Fry BN (2007). Molecular mimicry in campylobacter jejuni: Role of the lipo-oligosaccharide core oligosaccharide in inducing anti-ganglioside antibodies. FEMS Immunology \& Medical Microbiology, 50 (1): 27-36. DOI:https://doi.org/ 10.1111/j.1574-695X.2007.00225.x.

Pumbwe L, and Piddock LJ (2002). Identification and molecular characterisation of cmeb, a campylobacter jejuni multidrug efflux pump. FEMS Microbiology Letters, 206 (2): 185-189. DOI: https://doi.org/10.1111/j.1574-6968.2002.tb11007.x.

Schulze F, Bagon A, Müller W, and Hotzel H (2006). Identification of campylobacter fetus subspecies by phenotypic differentiation and pcr. Journal of clinical microbiology, 44 (6): 2019-2024. DOI:https://doi.org/ 10.1128/JCM.02566-05. 
Shippy DC, and Fadl AA (2014). Trna modification enzymes gida and mnme: Potential role in virulence of bacterial pathogens. International journal of molecular sciences, 15(10): 18267-18280. DOI:https://doi.org/ 10.3390/ijms151018267 .

Sircar R, Borbat PP, Lynch MJ, Bhatnagar J, Beyersdorf MS, Halkides CJ, Freed JH, and Crane BR (2015). Assembly states of flim and flig within the flagellar switch complex. Journal of molecular biology, 427 (4): 867-886. DOI:https://doi.org/ 10.1016/j.jmb.2014.12.009.

Sopwith W, Birtles A, Matthews M, Fox A, Gee S, Painter M, Regan M, Syed Q and Bolton E (2006). Campylobacter jejuni multilocus sequence types in humans, northwest england, 2003-2004. Emerging infectious diseases, 12 (10): 1500. DOI:https://doi.org/ 10.3201/eid1210.060048.

Szurmant H, Bunn MW, Cannistraro VJ, and Ordal GW (2003). Bacillus subtilis hydrolyzes chey-p at the location of its action, the flagellar switch. Journal of Biological Chemistry, 278 (49): 48611-48616. DOI:https://doi.org/10.1074/jbc.M306180200.

Taylor D, Ng L, and Lior H (1985). Susceptibility of campylobacter species to nalidixic acid, enoxacin, and other DNA gyrase inhibitors. Antimicrobial agents and chemotherapy, 28 (5): 708-710. DOI:https://doi.org/ 10.1128/aac.28.5.708.

Terahara N, Krulwich TA, and Ito M (2008). Mutations alter the sodium versus proton use of a bacillus clausii flagellar motor and confer dual ion use on bacillus subtilis motors. Proceedings of the national academy of sciences, 105(38): 14359-14364. DOI: https://doi.org/ 10.1073/pnas.0802106105 .

Thakur S, Zhao S, McDermott PF, Harbottle H, Abbott J, English L, Gebreyes WA and White DG (2010). Antimicrobial resistance, virulence, and genotypic profile comparison of campylobacter jejuni and campylobacter coli isolated from humans and retail meats. Foodborne pathogens and disease, 7 (7): 835-844. DOI:https://doi.org/ 10.1089/fpd.2009.0487.

Tikhonova EB, Devroy VK, Lau SYand Zgurskaya HI (2007). Reconstitution of the escherichia coli macrolide transporter: The periplasmic membrane fusion protein maca stimulates the atpase activity of macb. Molecular microbiology, 63(3): 895-910. DOI: https://doi.org/10.1111/j.1365-2958.2006.05549.x.

Tremblay C, and Gaudreau C (1998). Antimicrobial susceptibility testing of 59 strains of campylobacter fetus subsp. Fetus. Antimicrobial agents and chemotherapy, 42(7): 1847-1849. DOI: https://doi.org/ 10.1128/AAC.42.7.1847.

Tremblay C, Gaudreau C and Lorange M (2003). Epidemiology and antimicrobial susceptibilities of 111 campylobacter fetus subsp. Fetus strains isolated in quebec, canada, from 1983 to 2000. Journal of clinical microbiology, 41 (1): 463-466. DOI: https://doi.org/ 10.1128/jcm.41.1.463-466.2003.

Truyers I, Luke T, Wilson D, and Sargison N (2014). Diagnosis and management of venereal campylobacteriosis in beef cattle. BMC veterinary research, 10(1): 280. DOI: https://doi.org/10.1186/s12917-014-0280-x.

van der Graaf-van Bloois L, Miller WG, Yee E, Gorkiewicz G, Forbes KJ, Zomer AL, Wagenaar JA and Duim B (2016). Campylobacter fetus subspecies contain conserved type iv secretion systems on multiple genomic islands and plasmids. PloS one, 11(4). DOI: https://doi.org/10.1371/journal.pone.0152832.

Vasquez L, Ball L, Bennett B, Rupp G, Ellis R, Olson JD, and Huffman MH (1983). Bovine genital campylobacteriosis (vibriosis): Vaccination of experimentally infected bulls. American journal of veterinary research, 44 (8): 1553-1557. Available at: https://europepmc.org/article/med/6194723.

Wangroongsarb P, Jittaprasatsin C, Suwannasing S, Suthivarakom K, and Khamthalang T (2011). Identification of genus campylobacter and four enteropathogenic campylobacter species by pcr. Journal of Tropical Medicine and Parasitology, 34: 1729. Available at: www.ptat.thaigov.net.

Washington J, and Wood G (1995). Antimicroibial susceptibility tests: Dilution and disc diffusion methods. Manual of clinical microbiology.PP.1327-1331. Available at: https://pubmed.ncbi.nlm.nih.gov/5325707/.

Wieczorek K and Osek J (2013). Antimicrobial resistance mechanisms among campylobacter. BioMed research international, Article ID 340605, pp. 1-12. DOI: https://doi.org/10.1155/2013/340605.

Wieczorek K, Wołkowicz T and Osek J (2018). Antimicrobial resistance and virulence-associated traits of campylobacter jejuni isolated from poultry food chain and humans with diarrhea. Frontiers in microbiology, 9: 1508. DOI: https://doi.org/10.3389/fmicb.2018.01508.

Worby CA, Mattoo S, Kruger RP, Corbeil LB, Koller A, Zekarias B, Lazar C, and Dixon JE (2009). The fic domain: Regulation of cell signaling by adenylylation. Molecular cell, 34 (1): 93-103. DOI: https://doi.org/ 10.1016/j.molcel.2009.03.008 .

Yan M, Sahin O, Lin J, and Zhang Q (2006). Role of the cmeabc efflux pump in the emergence of fluoroquinolone-resistant campylobacter under selection pressure. Journal of Antimicrobial Chemotherapy, 58 (6): 1154-1159. DOI: https://doi.org/ $\underline{10.1093 / \mathrm{jac} / \mathrm{dkl} 112 .}$.

Yuan J, Branch RW, Hosu BG, and Berg HC (2012). Adaptation at the output of the chemotaxis signalling pathway. Nature, 484 (7393): 233-236. DOI: https://doi.org/ 10.1038/nature10964. 